\title{
Inkjet Printing of Ultrawideband (UWB) Antennas on Paper-Based Substrates
}

\author{
George Shaker, Student Member, IEEE, Safieddin Safavi-Naeini, Member, IEEE, Nagula Sangary, Member, IEEE, \\ and Manos M. Tentzeris, Fellow, IEEE
}

\begin{abstract}
For the first time, we demonstrate the feasibility of realizing ultrawideband antennas through ink-jetting of conductive inks on commercially available paper sheets. The characterization of the conductive ink as well as of the electrical properties of the paper substrate are reported for frequencies up to $10 \mathrm{GHz}$. This letter is one step further toward the development of low-cost, environment-friendly conformal printed antennas/electronics for ad hoc wireless sensor networks operating in rugged environments.
\end{abstract}

Index Terms-Inkjet printing, low-cost "green" electronics, ultrawideband (UWB) antennas, wireless sensor networks.

\section{INTRODUCTION}

$\mathbf{U}$ LTRAWIDEBAND (UWB) RF technologies are increasingly used as a means of short-range, high-bandwidth communications utilizing very low power levels spreading the transmitted signal over a significantly large portion of the radio spectrum, commonly between $3.1-10.5 \mathrm{GHz}$. Interestingly, numerous recent applications of UWB radios target sensor data collection, precision localization, and tracking applications [1]. Such applications necessitate the deployment of a large number of UWB antennas to meet system requirements. To this end, it is important to keep the cost per antenna as low as possible to maintain an adequate operational cost for such UWB systems. A quick look at the most common techniques for the fabrication of printed UWB antennas reveals that photolithography has been the most dominant technology. However, this method involves multiple steps including etching, masking, and electroplating, thus being a time-consuming, labor-intensive, and expensive process. In addition, since the solvent used in the etching process is corrosive, the choice of substrates is limited. Moreover, the photolithography process generates high volumes of hazardous waste that are environmentally detrimental. An alternative technique is thus needed.

Manuscript received December 11, 2010; revised January 09, 2011; accepted January 11, 2011. Date of publication January 17, 2011; date of current version March 14, 2011. This work was supported by NSERC CGS-FSS, NSF ECS0801798, the Georgia Electronic Design Center, and the Interconnect Focus Center (IFC-SRC).

G. Shaker is with University of Waterloo, Waterloo, ON N2L 3G1, Canada, and also with the Georgia Institute of Technology, Atlanta, GA 30332 USA (e-mail: gshaker@uwaterloo.ca).

S. Safavi-Naeini and N. Sangary are with the University of Waterloo, Waterloo, ON N2L 3G1, Canada (e-mail: safavi@uwaterloo.ca).

M. M. Tentzeris is with the Department of Electrical Engineering, Georgia Institute of Technology, Atlanta, GA 30332 USA (e-mail: etentze @ gatech.edu).

Color versions of one or more of the figures in this letter are available online at http://ieeexplore.ieee.org.

Digital Object Identifier 10.1109/LAWP.2011.2106754

\section{MATERIAL CHARACTERIZATION}

\section{A. Printing Setup}

In this letter, we propose the utilization of inkjet printing of conductive inks on paper sheets to realize low-cost, environment-friendly UWB antennas up to frequencies above $10 \mathrm{GHz}$. Paper possesses a number of intriguing attributes that makes it amenable for low-cost "green" electronics. It is cellulose in nature, thus considered as a renewable resource. Additionally, it can be easily processed in a reel-to-reel fashion, enabling low-cost manufacturing solutions. Driven by the fact that it is challenging/cost-inefficient to apply photolithography techniques to paper, inkjet printing of conductive particles provides a promising solution [2], [3]. It is widely known that one of the most common methods of digital printing is through inkjet printers, almost ubiquitous in home or office applications. Notably, inkjet printing has been recently enhanced with the capability of printing new functional materials, such as conductive and carbon nanotube CNT-based inks, leading to an increased deployment in printed electronics, such as flexible displays, RFIDs, sensors, solar panels, fuel cells, batteries, and most recently in antennas [4]-[8].

Inkjet printing for RF applications is a challenging endeavor, where precise control of the achieved conductivity and surface roughness are required. In general, there are two critical factors that affect the print quality: the ink properties and the settings of the printing system itself. The most notable ink properties to observe are viscosity, surface tension, and dispersion stability (printing with high contact angle, high viscosity, and high-tension ink produces smaller sized dot patterns). The most crucial settings of the printing parameters include the volume of the jetted ink, the traveling velocity of the ejected droplet, the gap distance between each droplet, the printing frequency, the temperature of the jetted ink, the temperature of the substrate, and the sintering/curing mechanism performed on the printed structures.

In this letter, we have utilized the DMP2800 inkjet printer, which is a tabletop printer available from Dimatix, Inc. (www. dimatix.com), shown in Fig. 1. To ensure good RF properties, an in-house recipe was developed considering all of the aforementioned parameters. In all prints, the utilized Dimatix $1 \mathrm{pL}$ cartridges (DMC-11601) were kept at a distance of $0.5 \mathrm{~mm}$ from the surface of paper on a DMP-2800 printer. The printer head was adjusted to achieve a print resolution of $2540 \mathrm{dpi}$, which ensures good RF conductivity up to several gigahertz. Cabot conductive ink CCI-300 (www.cabot-corp.com) was then jetted at a temperature of $40^{\circ} \mathrm{C}$, while the paper substrate was maintained 


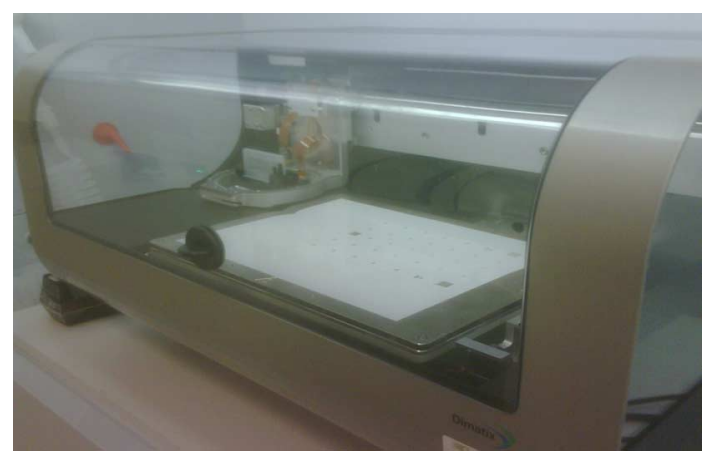

Fig. 1. Tabletop inkjet printer used in this letter.

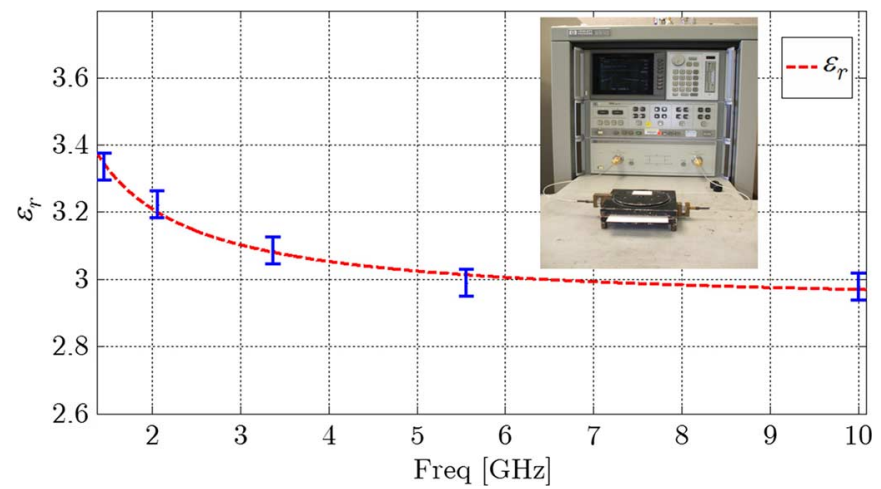

Fig. 2. Characterization of the paper material through the split-ring resonator method.

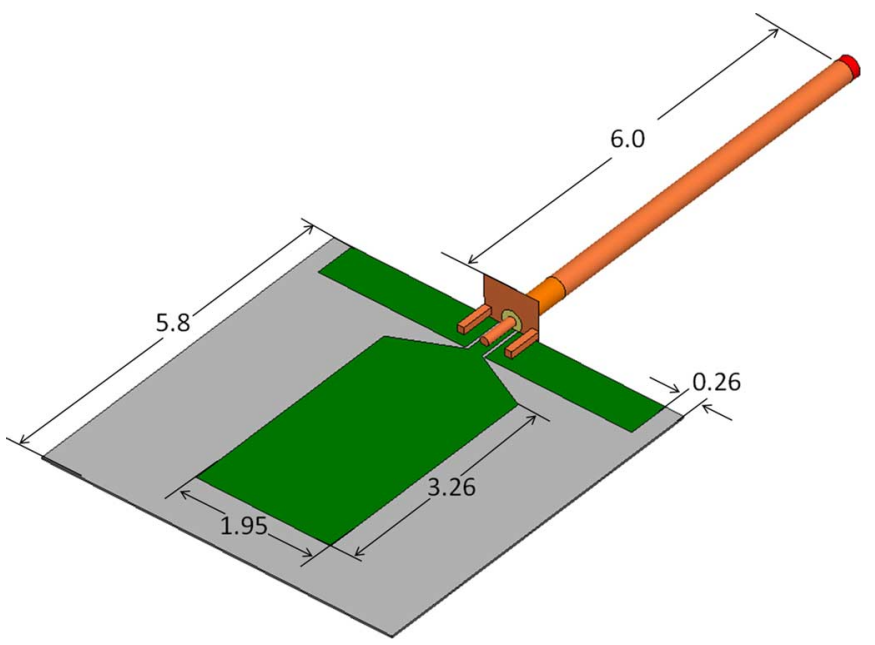

Fig. 3. Numerical model for the printed antenna.

at $60^{\circ} \mathrm{C}$. Each printed structure was then cured in a thermal oven for $2 \mathrm{~h}$ at $120^{\circ} \mathrm{C}$.

\section{B. Paper Characterization}

The properties of the benchmarking paper substrates were studied in the UWB frequency range through the use of the split-post dielectric resonator technique [9], [10]. Several cavities covering the band from 1 to $10 \mathrm{GHz}$ were utilized. Each blank paper sample was cured first in a thermal oven for $2 \mathrm{~h}$ at $120^{\circ}$ to mimic the curing process of the printed ink. The results for the extracted relative permittivity of the 10-mil-thick cured

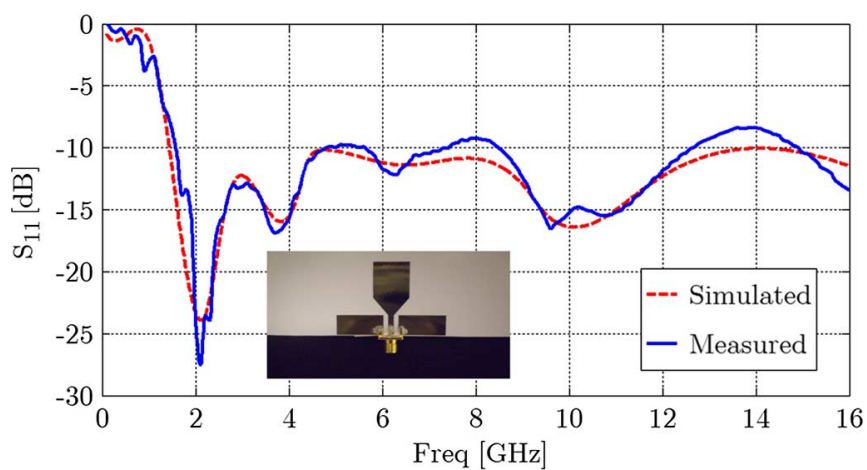

Fig. 4. Simulated and measured performance of the antenna.

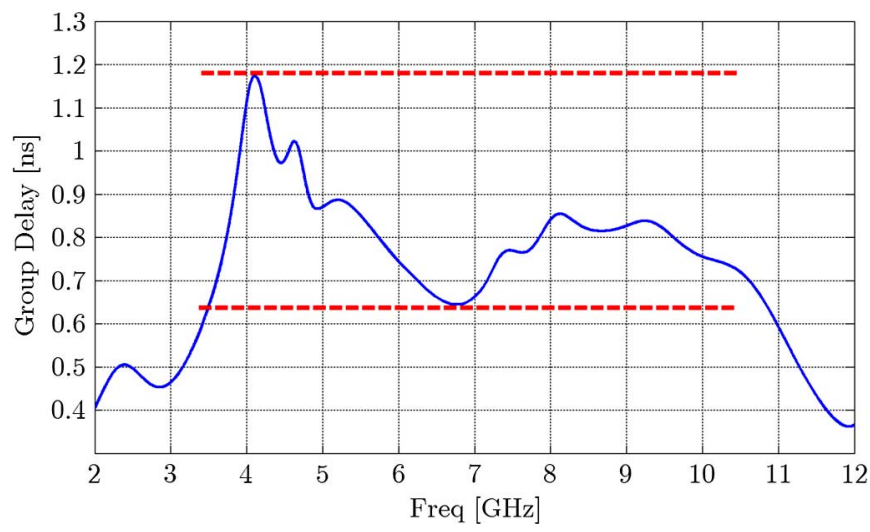

Fig. 5. Resulting group delay of the antenna.

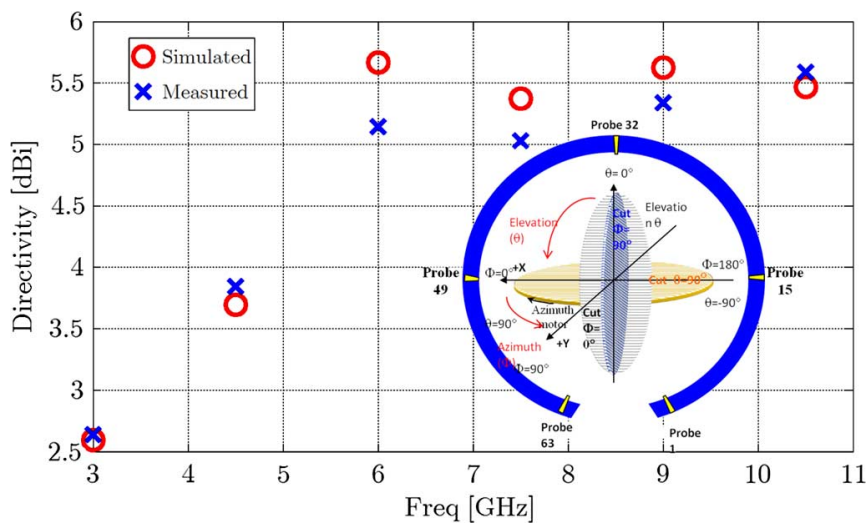

Fig. 6. Measured and simulated directivity of the antenna.

paper are shown in Fig. 2. The measured dielectric loss tangent values were bounded between 0.06 and 0.07 for the whole frequency range.

\section{Ink Characterization}

The conductivity of the printed conductive ink was studied through the use of the Signatone Four Point Probe (www.signatone.com). To ensure good RF conductivity, three layers of ink were printed and then treated in a thermal oven as described earlier. The resulting ink thickness was measured using the Wyko profilometer (www.veeco.com). The resulting thickness was around $3 \mu \mathrm{m}$ with a consistent measured $\mathrm{dc}$ conductivity in the range $9 \times 10^{6}[\mathrm{~S} / \mathrm{m}]-1.1 \times 10^{7}[\mathrm{~S} / \mathrm{m}]$. 
TABLE I

Measured Radiation Patterns at Select in-Band Frequencies ( - For $x z$ Plane and -- for $y z$ Plane)

(1)

\section{ANTENNA DESIGN}

To investigate the applicability of inkjet-printed paper-based technology for the realization of UWB structures, a planar UWB monopole was adopted in this letter for its simplicity [11], [12] as a proof-of-concept geometry. The design was optimized through full-wave finite-difference time-domain (FDTD) simulations using a commercially available solver from SPEAG (www.semcad.com). The simulations incorporated the actual ink thickness along with the frequency-dependent permittivity of the paper substrate. The antenna was printed on a paper sheet following the outlined guidelines. The paper sample was cut to a square slice of $58 \times 58 \mathrm{~mm}^{2}$ to form the overall antenna [computer-aided design (CAD) model shown in Fig. 3]. A model for a long piece of coaxial cable was used in simulations to mimic the actual piece used in measurements. This usually helps in modeling the common-mode currents flowing on the feeding cable, and typically results in better correlation between the measured and simulated pattern/gain values. It is noteworthy to observe that such an antenna is fully conformal.

The Agilent PNA-X network analyzer (N5245A) was used for the scattering parameters measurements. Fig. 4 shows good agreement between the simulated versus the measured responses at the input port of the antenna up to $16 \mathrm{GHz}$. The resulting group delay is plotted in Fig. 5. For proper UWB operation, it is important to keep the variation in the group delay value as low as possible so as not to distort the trans$\mathrm{mitted} /$ received signals. This proof-of-concept prototype shows an acceptable variation in the group delay up to a maximum of $0.6 \mathrm{~ns}$.

\section{FAR-Field MEASUREMENTS}

The radiation pattern was measured using Satimo's Stargate 64 Antenna Chamber measurement system. The NIST Calibrated SH8000 Horn Antenna was used for the calibration of the measured radiation pattern at the selected in-band frequencies $(3,4.5,6,7.5,9$, and $10.5 \mathrm{GHz})$. The prototype antenna was placed in the $y z$ plane as outlined in Fig. 6, with the $\mathrm{x}$-axis normal to the plane of the antenna. The measured radiation pattern at different elevation angles is shown in Table I. The resulting pattern is almost uniform (omnidirectional) for the selected in-band frequencies, which is ideal for many UWB applications. The antenna efficiency was better than $80 \%$ throughout the whole band. Fig. 6 shows good correlation between the measured and simulated directivity values. Better correlation can be achieved through accurate modeling of the feeding cable length, and its actual mounting configuration, in addition to proper choking of the common mode currents flowing on the cable. 


\section{CONCLUSION}

This letter illustrates the feasibility of realizing efficient ultra-ideband antennas through ink-jetting of conductive inks on commercially available paper sheets up to frequencies above $10 \mathrm{GHz}$. This work could potentially pave the way toward the next-generation of low-cost, environment-friendly ubiquitous UWB sensor networks.

\section{ACKNOWLEDGMENT}

The authors would like to acknowledge the assistance received in material characterization from Dr. S. Kim and Dr. J. R. Baker-Jarvis with the Electromagnetics Division, National Institute of Standards and Technology (NIST), Boulder, CO. The authors would also like to acknowledge the assistance received in radiation pattern measurements from A. Rida and K. Rutowski with Satimo, Kennesaw, GA.

\section{REFERENCES}

[1] J. Zhang, P. Orlik, Z. Sahinoglu, A. F. Molisch, and P. Kinney, "UWB systems for wireless sensor networks," Proc. IEEE, vol. 97, no. 2, pp. 313-331, Feb. 2009.

[2] J. Mei, M. Lovell, and M. Mickle, "Formulation and processing of novel conductive solution inks in continuous inkjet printing of 3-D electric circuits," IEEE Trans. Electron. Packag. Manuf., vol. 28, no. 3, pp. 265-273, Jul. 2005.
[3] S. Magdassi, The Chemistry of Inkjet Inks. Singapore: World Scientific, 2009.

[4] J. Siden, M. Fein, A. Koptyug, and H. Nilsson, "Printed antennas with variable conductive ink layer thickness," Microw., Antennas Propag., vol. 1, no. 2, pp. 401-407, Apr. 2007.

[5] L. Yang, A. Rida, R. Vyas, and M. Tentzeris, "RFID tag and RF structures on a paper substrate using inkjet-printing technology," IEEE Trans. Microw. Theory Tech., vol. 55, no. 12, pp. 2894-2901, Dec. 2007.

[6] M. Mantysalo and P. Mansikkamaki, "An inkjet-deposited antenna for $2.4 \mathrm{GHz}$ applications," Int. J. Electron. Commun., vol. 63, no. 1, pp. 31-35, Jan. 2009.

[7] L. Yang, R. Zhang, D. Staiculescu, C. Wong, and M. Tentzeris, "A novel conformal RFID-enabled module utilizing inkjet-printed antennas and carbon nanotubes for gas-detection applications," IEEE Antennas Wireless Propag. Lett., vol. 8, pp. 653-656, 2009.

[8] L. Yang, G. Orecchini, G. Shaker, H. Lee, and M. Tentzeris, "Battery-free RFID-enabled wireless sensors," in Proc. IEEE Int. Microw. Symp., 2010, pp. 1528-1531.

[9] M. D. Janezic and J. Baker-Jarvis, "Full-wave analysis of a split-cylinder resonator for nondestructive permittivity measurements," IEEE Trans. Microw. Theory Tech., vol. 47, no. 10, pp. 2014-2020, Oct. 1999.

[10] J. Krupka, K. Derzakowski, A. Abramowicz, B. Riddle, J. Baker-Jarvis, R. N. Clarke, and O. C. Rochard, "Bounds on permittivity calculations using the TE01 $\delta$ dielectric resonator," in Proc. 14th Int. Conf. Microw., Radar Wireless Commun., 2002, pp. 394-396.

[11] M. Peyrot-Solis, G. Galvan-Tejada, and H. Jardon-Aguilar, "A novel planar UWB monopole antenna formed on a printed circuit board," Microw. Opt. Technol. Lett., vol. 48, no. 5, pp. 933-935, 2006.

[12] Z. N. Low, J. H. Cheong, and C. L. Law, "Low-cost PCB antenna for UWB applications," IEEE Antennas Wireless Propag. Lett., vol. 4, 2005. 